\title{
Low Profile Stretch Sensor for Soft Wearable Robotics
}

\author{
S. Sareh, Member, IEEE, and Y. Noh
}

\begin{abstract}
- this paper presents a low profile stretch sensor for integration into soft structures, robots and wearables. The sensor mechanism uses a single piece of highly flexible and light weight optical fibre and is based on the notion that bending an optical fibre modulates the intensity of the light transmitted through the fibre, a technique often referred as macrobending light loss. In this arrangement, the optical fibre originates from sensor's electronic unit, passes through a stretchable encasing structure in a macrobend pattern, and then loop back to the same unit resulting in a simplified electrical and optical design; the closed optical loop allows for no electronics at one end of the sensor making it safe for human robotics applications, and no optical interference with the external environment eliminating the need for complex conditioning circuitries. Of particular interest of the soft robotics community, the ability of this custom macrobend stretch sensor to flexibly adapt its configuration allows preserving the inherent softness and compliance of the robot which it is installed on. Our experimental results indicate that the optical fibre's bending radius is the dominant design parameter for sufficiently complex patterns, a finding that can facilitate generalisation of the sensing methods across different scales. The measurement performance of the mechanism and its impact on the stiffness of the encasing structure is benchmarked against a custom calibration and testing system.
\end{abstract}

\section{INTRODUCTION}

Manufacturing of wearable robots using soft materials and actuation components [1-4] promises great potential particularly in the context of safe human-robot interaction [5,6], ergonomics and comfortableness [7]. However, it is faced with intriguing engineering challenges with respect to configuration and control [8]. In order to accurately control the robot motion, an appropriate sensing technology should be integrated into the robot structure. As an important requirement for maintaining the advantages of soft robots, the integrated sensor devices must preserve the softness of the robot structure [9]. Hence, development of novel sensors made out of soft or flexible materials is an important topic of research within the community. Such sensors are expected to provide useful information on the robot's shape that can explain the motion patterns of the person wearing it. The information can subsequently be used to control the robot and to create a better understanding of the environment.

S. Sareh is with Design Robotics, School of Design, Royal college of Art, London, UK. Y. Noh is with the Department of Biomedical Engineering, King's College London. (corresponding author: sina.sareh@rca.ac.uk).

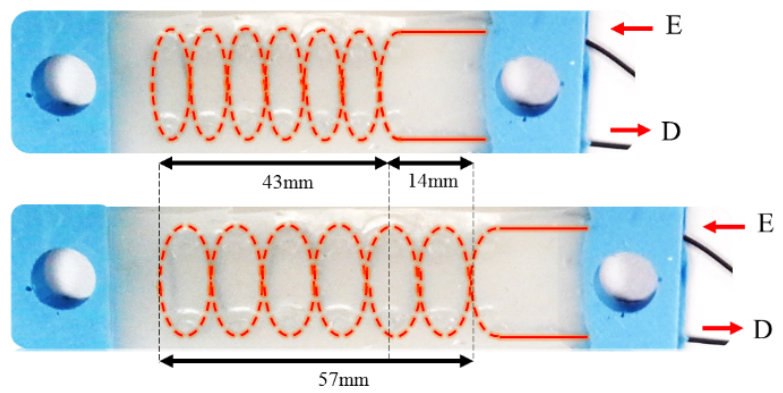

Figure 1. The proposed stretch sensor based on a single piece of optical fibre. Note that the superimposed dashed line in red shows the path for the optical fibre integrated inside the silicone structure. ' $\mathrm{E}$ ' and ' $\mathrm{D}$ ' indicate the connections to the light emitter and detector, respectively.

The complex motion patterns generated by these robots have been mainly tracked through incorporation of vision systems [10] and electromagnetic tracking [11]. However the visual techniques are often restricted with visual occlusion and electromagnetic tracking is subject to magnetic field distortions and have limitations with regards to mobility of the magnetic field generation system.

An effective approach in soft robotic shape sensing is the integration of multiple stretch sensors into the robot's body, in a strategic way. The information on the change of length at different locations can be then translated into appropriate shape information for use in robot control applications $[9,11]$.

One of the best examples of off-the-shelf stretch sensors is resistive sensors based on conductive rubber materials (Images Scientific Instruments Inc, USA). Although, these sensors can be used for measuring the length via simple conditioning circuits, they suffer from large relaxation times and hysteresis [12]. Stretch sensors based on dielectric elastomers, supplied by StretchSense Ltd (New Zealand) and Danfoss PolyPower A/S (Denmark), are able to accurately measure the stretched length by a near linear voltage-length relationship. However, decoupling between different modes of sensing, e.g. pressure and stretch modes, is complex [13].

Fibre optic sensing systems work based on the modulation of the optical characteristics of the light transmitted through the optical fibre, including light intensity [14-19], wavelength [20], and polarization [21]. Light intensity modulation, modulating the intensity of the light transmitted via the fibre, has been employed in different configurations for pose and force sensing in soft robotics [9,15-19]. Fibre Bragg Grating (FBG) sensors have been used for shape sensing in continuum robots [22]. However, FBG systems are highly sensitive to 
strain conditions. If they are subjected to a non-uniform strain field, the strain compensation is complex [23].

Bending an optical fibre is an approach for light intensity modulation. Macrobend light loss happens by sufficient bending of the optical fibre beyond the point at which the critical angle for light reflection is exceeded [24]. The macrobending of an optical fibre connected to a light source can occur at multiple locations along the length of the fibre and lead to light loss that can be measured using a light detector [25]. Macrobend stretch sensors were proposed different configurations including semicircular (half turn), circular (one turn), and figure-of-eight [26] and were employed in a multi-layer configuration for plantar pressure and shear sensing [27] and for the development of a voltage sensor [28]. In this paper, we investigate the macrobendbased sensing approach for developing low profile stretch sensors.

The proposed stretch sensing system comprised of a fibre sensor mechanism; a lightweight and highly flexible optical fibre connected to a light emitter, passed through a stretchable encasing structure in a serpentine macrobend pattern, and then loop back to connect to a light detector, which is collocated with the emitter, as shown in Figure 1. As the sensor mechanism is stretched the amount of light transmitted between the optical emitter and detector changes, the change is measured using a FS-N11MN (Keyence, USA) fibre optic unit and translated into the respective change of length information.

\section{MATERIALS AND METHODS}

In this section, we describe our approach in designing the stretch sensor anchored on characterization of macrobendinduced light loss experiments. Through this study, we aim to characterize the main parameters of the proposed stretch sensors: the bend fibre radius and the number of turns. The definition of an optical fibre turn with bend fibre radius $r$ is illustrated in Figure 2 (a,b) [9].

\section{A) Measurement of macrobend-induced light loss}

In order to characterize the fibre macrobend, precise bending of the optical fibre is required. The industry standard IEC 60793-1-47 (measurement methods and test procedures: macrobending) recommends two methods for the bending of optical fibres for macrobend loss measurement including fibre winding, e.g. using mandrels, and quarter circle bends, e.g. using guiding groove structures on a flat surface (Figure $2(c, d))$. Whilst the former is the preferred way of bending for relatively large number of fibre turns, the later is more suitable for bending optical fibres in short lengths with relatively fewer bends.

In an experiment the planar bend patterns were approximated using 3D winded patterns. This experiment uses both rigs, grooves and mandrels, and is aimed to identify the difference in macrobend loss when equal number of turns are produced

\footnotetext{
${ }^{1}$ In this study, the SH1001-1.0 Super Eska ${ }^{\mathrm{TM}}$ Polyethylene jacketed optical fiber cord (Mitsubishi Rayon Co., Ltd., Japan) with a core
}

through winding around a mandrel (Figure 2(c)) versus forming inside successive guiding grooves (Figure 2(d)). The experiment was performed for number of turns $\mathrm{N}=\{1,2,3\}$. The experimental results imply that the sensing voltage depends on the bending radius and the number of turns in both cases, i.e. there are approximately equal sensing values (maximum difference of 3\%) for two successive half turn using guiding grooves and a turn using mandrels. Therefore, we can calculate the macrobend loss in complex helical or 2D patterns of mutually-tangent circular arcs only through counting the number of turns and associated radii.

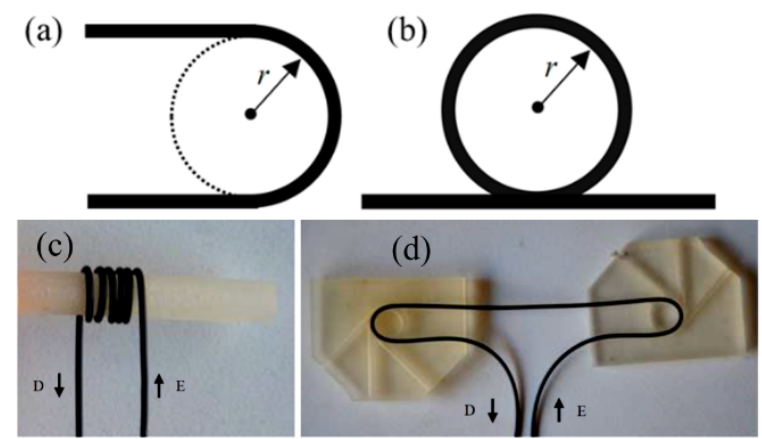

Figure 2. The definition of the optical fibre turn: a half-turn (a) vs. a turn (b), with bend fibre radius $r$. Note that the bend fibre radius is measured from the inside curvature, (c) winding the fibre around a mandrel (three turns), and (d) a piece of optical fibre is precisely bent using two guiding groove structures on a flat surface (a total bending of one turn) [9].

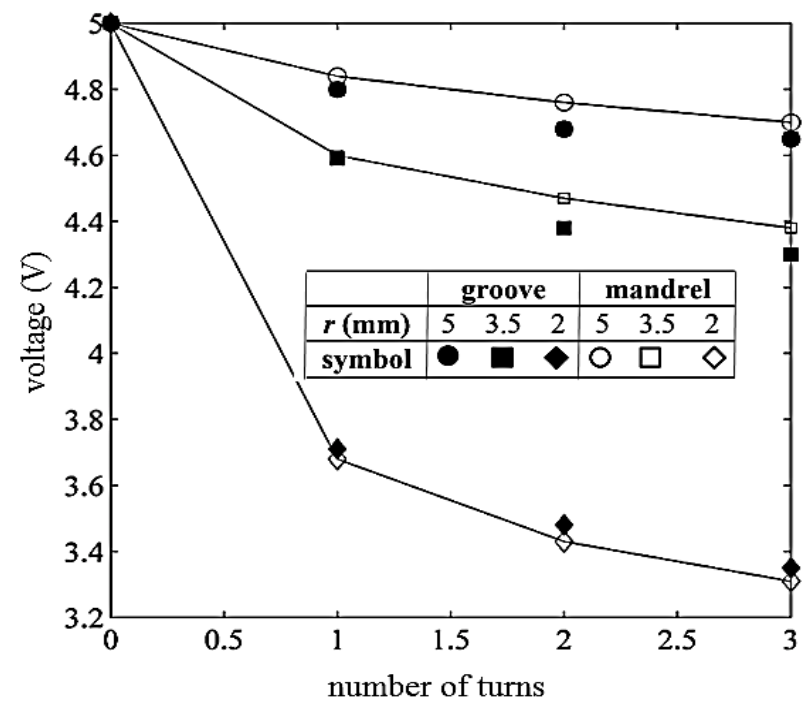

Figure 3. The experimental data which indicates that bending the fibre through the two above-mentioned approaches (using mandrels and guiding grooves) achieves virtually the same results (voltage values) [9].

In this study, we focus on designing serpentine (snake-like) stretch patterns requiring large number of optical fibre bends. Hence, a set of custom mandrels for the bending and winding of optical fibres were prepared ${ }^{1}$. When the optical fibre is

diameter of $217-263 \mu$ and weight of $0.74 \mathrm{~g} / \mathrm{m}$ has been used for experimentation. The mandrels were designed in SolidWorks 3D 
bent, the intensity of the modulated light is measured using the FS-N11MN fibre optic unit, which provides a pair of light emitter and light detector and converts the light intensity to voltage. In order to characterize the macrobend loss, mandrels of various cross-section radii $r=\{2,2.5,3,3.5,4,4.5,5,5.5$, $6,6.5,7\} \mathrm{mm}$ for winding multiple turns $\mathrm{N}=\{1, \ldots, 10\}$ of optical fibres were used. It was observed that increasing the number of fibre turns leads to a reduction in the intensity of the light detected by FS-N11MN and the respective sensing voltage (Figure 4(a)). Note that the measurements of the voltage were taken every half a turn, hence we have 21 measurement points per radius. It can be seen that, there is a clear elbow in each constant radius graph where the number of turns is 5 . After this point, the rate of reduction in voltage values is negligible, e.g. a maximum change of $4 \%$ in the voltage values was measured whilst increasing the number of turns from $\mathrm{N}=5$ to $\mathrm{N}=10$ across different radii. In order to further explain this observation, the standard deviation (SD) of the last 11 samples of voltage at each radius was calculated and then divided by the standard deviation of the whole 21 samples at each radius to result in a normalized standard deviation expressed as [9],

$$
\frac{\text { SD of last } 11 \text { voltage samples at constant radius } r}{\text { SD of all voltage samples at constant radius } r}=\frac{\sqrt{\frac{\sum_{i=11}^{21}\left(v_{i, r}-\eta_{r}\right)^{2}}{11}}}{\sqrt{\frac{\sum_{i=0}^{21}\left(v_{i, r}-\mu_{r}\right)^{2}}{20}}}
$$

where $v_{i, r}$ is the voltage value corresponding to the sample number $i=\{1, \ldots, 21\}$ and the bending radii $r=\{0.5,1,1.5,2$, $2.5,3,3.5,4,4.5,5,5.5,6,6.5,7\}$. The $\eta_{r}$ and $\mu_{r}$ represent average voltage values at constant radius $r$,

$$
\eta_{r}=\frac{1}{11} \sum_{i=11}^{21} v_{i, r}, \mu_{r}=\frac{1}{21} \sum_{i=0}^{21} v_{i, r}
$$

Figure 4(b) shows that the ratio of absolute deviation of the last 11 samples and the standard deviation of all 21 samples significantly decrease by increasing the bend fibre radius. Note that, in Figure 4(c) the absolute standard deviation of the last 11 voltage samples at each radius is shown, which is less than $0.04 \mathrm{~V}$. Therefore, the dependence of the voltage to the number of turns can be assumed negligible when the number of turns is equal or greater than 5. This assumption simplifies designing sensing systems based on macrobend loss, taking into account the bend radius as the only significantly influencing design parameter. Hence, in this study, we focus on the design and implementation of a stretch sensor with number of turns equal or greater than 5 .

In general, the light loss in an optical fibre occurs when the radius of bending is below a specific threshold called the Minimum Bending Radius (MBR). This value is a function of the material properties, thickness, and also the number of turns [29, 30]. The MBR for a quarter turn of the SH1001-1.0 Super Eska ${ }^{\mathrm{TM}}$ Polyethylene jacketed optical fibre used in this study is $5 \mathrm{~mm}$, according to its datasheet. It was

CAD software (SolidWorks Corp.) and fabricated from Visijet EX200 via a Projet HD 3000 3-D production system. experimentally observed that a quarter bend can produce 0.1 $\mathrm{V}$ drop in the sensing voltage. Clearly, increasing the number of turns causes drop in sensing voltage and, hence, raises the value of the MBR. It is worth reiterating that, in this study, we aim at designing macrobend sensors with $\mathrm{N} \geq 5$. In an experiment we observed that, a $0.1 \mathrm{~V}$ voltage drop happens by bending under the radius of $6.5 \mathrm{~mm}$ when $\mathrm{N} \geq 5$. Hence, the value of MBR is $6.5 \mathrm{~mm}$ for the proposed stretch sensor.

\section{B) Macrobend stretch sensor design}

The design of the macrobend stretch sensor has considered the following criteria: (1) it should be highly compact at compression, and stretchable to a particular length required by the actuator it is coupled with to measure its length change.

(2) in order to keep sensor optoelectronics away from the sensing site, the fibre should be looped to place the light emitter and detector next to each other, at one end of the sensor.

The fabrication of the sensor started by designing and fabrication a simple container that allow easy integration of the optical fibre in a serpentine form, followed by the insertion of the fibre and filling the container with Eco-flex 00-50 silicone rubber materials (Smooth-on Inc.). The combination was cured in room temperature.

Furthermore, the loopback design of the macrobend stretch sensor is illustrated in Figure 5(a) indicating locations of tip vertices, lateral vertices, and fibre cross-overs. It is important to note that during a full stretch of the sensor, the radius of curvature of lateral vertices are increased resulting in an increment in the light intensity, whilst the radius of curvature at the tip vertex is decreased generating an inverse effect on the light intensity value. Therefore, the voltage output is not valid if the bend fibre radius at the tip vertex is less than the MBR of the optical fibre $(r<6.5 \mathrm{~mm})$. In order to avoid this problem, the radius of curvature at the tip vertex of the sensor must be always kept above the MBR, so that this vertex is not used for sensing.

Three key phases of stretch sensing, each comprised of a chain of ellipsis where $a$ and $b$ represent semi major axes of each ellipse, are shown in Figure 5(a,b). Each ellipse has two lateral vertices that can be designed to produce certain levels of light loss at certain stretch levels of the sensor structure. The levels of light loss depend on how the bend fibre radius of lateral vertices is changing in relation with the MBR. We assume that the perimeter of each ellipse remains constant during the length change of the sensor structure, which means there is no exchange of fibre length at fibre cross-over locations during the operation of the sensor. Hence, given the number of ellipses $\mathrm{N}$ and the bend fibre radius of the balanced configuration ( $r$ balance), a unique sensor structure can be designed. Table 1 summarizes the impact of the choice of $r_{\text {balance }}$ on the compactness and stretchability of the sensor, suggesting that $b_{\max }=r_{\text {balance }}=$ MBR can be considered as a reasonable trade-off in the design of the sensor. Note that 
$b_{\max }$ is regarded as a measure for the stretchability of the sensor, also $w_{\max }$ increases with $b_{\max }$ and represents the maximum value of the semi major axis $b$. In an experiment we observed that the FS-N11MN voltage value is $1.35 \mathrm{~V}$ at a bend fibre radius of $0.5 \mathrm{~mm}$ (this value was measured by winding the fibre around a still needle with the diameter of $1 \mathrm{~mm})$. The $b=0$ cannot be implemented and tested using mandrels. Here, we consider $b_{\min }=0.5 \mathrm{~mm}$ to allow sufficient voltage margin with the radius range that cannot be tested through this approach, where $b_{\min }$ is the minimum value of the semi major axis $b$.

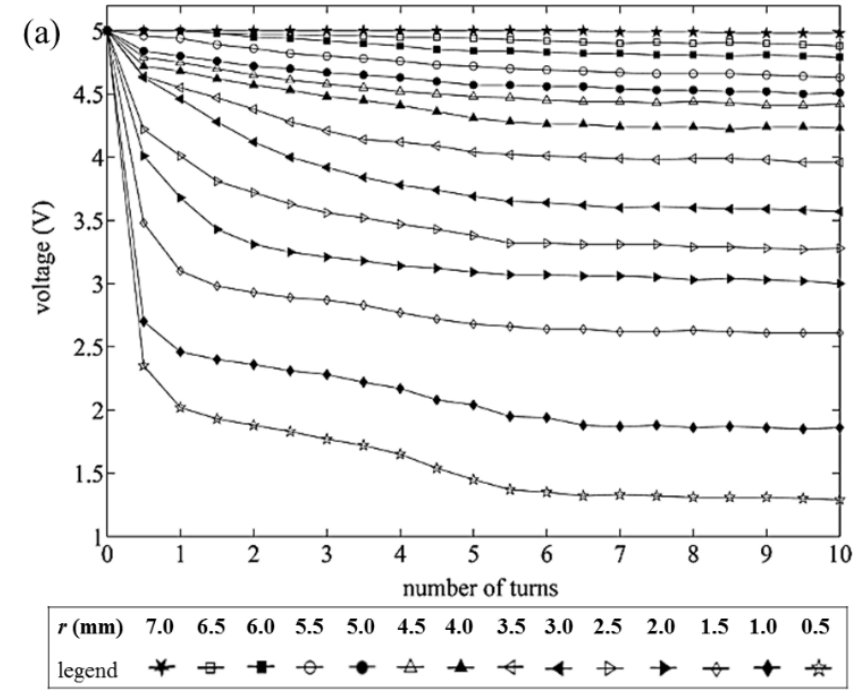

(b)
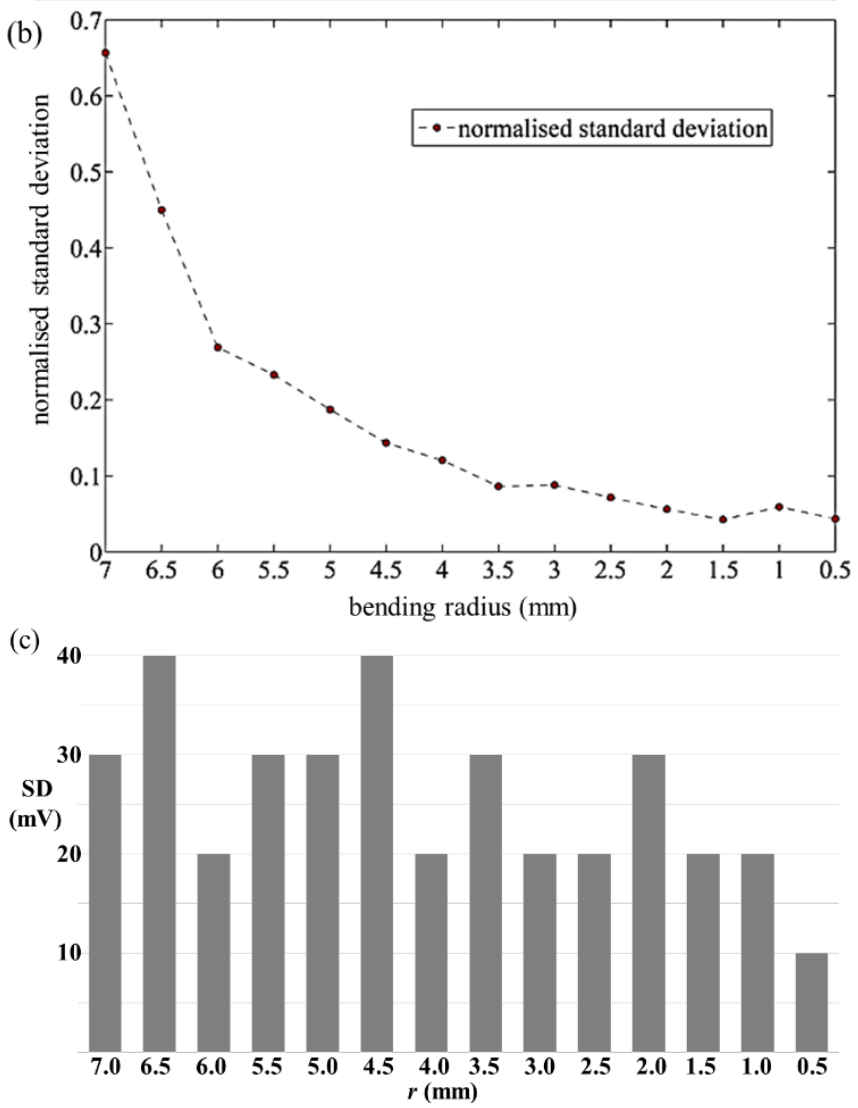

Figure 4. (a) The FS-N11MN voltage output as the number of macrobend turns around the mandrel is increased, (b) the radio of standard deviation of last 11 samples and the standard deviation of all 21 samples significantly decreases by increasing the bend fibre radius, (c) the absolute standard deviation of last 11 voltage samples at each bend fibre radius [9].

The design of stretch sensors requires information on the minimum and maximum stretch at these locations. Referring to the Figure 5(a), the number of ellipsis can be calculated through,

$$
N=\frac{L-2 \mathrm{~N} b}{\mathrm{f}}-1
$$

(a)

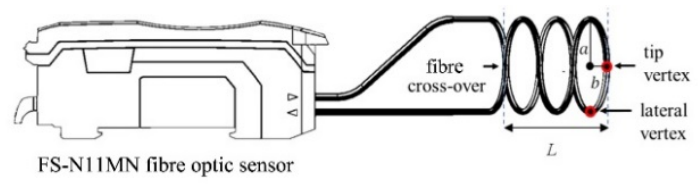

(b)

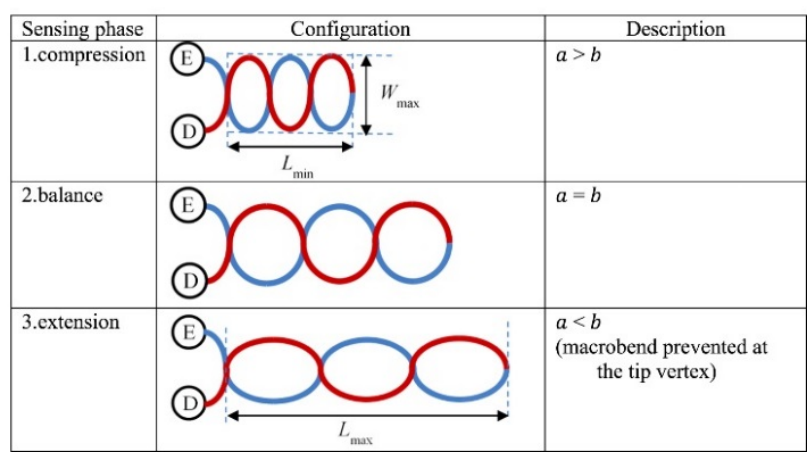

Figure 5. (a) The loopback configuration of the macrobend stretch sensor, (b) the three key phases of sensing including compression, balance, and extension [9].

In this study, the active length of the sensor is $L=$ $L_{\max }=57 \mathrm{~mm}, b=4.2 \mathrm{~mm}<b_{\max }=6.5 \mathrm{~mm}$, and $\mathrm{f}=1 \mathrm{~mm}$, and therefore, the number of ellipsis (fibre turns) can be computed as $\mathrm{N}=6$. Note that $\mathrm{N}=6$ is satisfying the optical condition $(\mathrm{N} \geq 5)$ for relying only on the radius of curvature in calculation of the macrobend loss, discussed in Figure 4(a) and 4(b).

\section{C) Sensor calibration and benchmarking}

In order to experimentally validate the sensor design, the sensor was mounted on a custom calibration system comprised of a motorised linear guide, a motion controller and a Nano17 6-axis F/T sensor. By stretching the sensor, the macrobend structure is expected to be continuously modified from highly compressed to the balanced configuration, ideally providing a sensing range of $1-5 \mathrm{~V}$ which is the measurable range by FS-N11MN fibre optic unit.

6(a) shows the experimental setup of a test where the stretch sensor was subjected to uniaxial elongation. The voltage output of the FS-N11MN fibre optic unit and the motor position were synchronously acquired during the test. This characterization test was run for 5 cycles and the sensor's voltage-length relationship and respective error values are presented in Figure $6(\mathrm{~b}, \mathrm{c})$. The maximum error of $2.8 \mathrm{~mm}$ can be due to possible latency caused by hysteresis, resulting from integration into the silicone structure. 
Table 1. The choices of the balance bend fibre radius $r_{\text {balance }}$ and their impact on stretchability and compactness of the sensor structure.

\begin{tabular}{|c|l|l|l|l|}
\hline \multicolumn{2}{|c|}{$\boldsymbol{r}_{\text {balance }}$} & $<$ & $=$ & $>$ \\
MBR & MBR & MBR \\
\hline \multirow{2}{*}{\begin{tabular}{c} 
Configuration \\
\cline { 2 - 5 }
\end{tabular}} & compression & yes & yes & yes \\
\cline { 2 - 5 } & balance & no & yes & yes \\
\hline extension & no & no & yes \\
\hline $\begin{array}{c}\text { Stretchability } \\
\left.\text { (measure: } \boldsymbol{b}_{\text {max }}\right)\end{array}$ & low & medium & high \\
\hline $\begin{array}{c}\text { Compactness } \\
\text { (measure: } \boldsymbol{w}_{\text {max }} \text { ) }\end{array}$ & high & medium & low \\
\hline
\end{tabular}

(a)
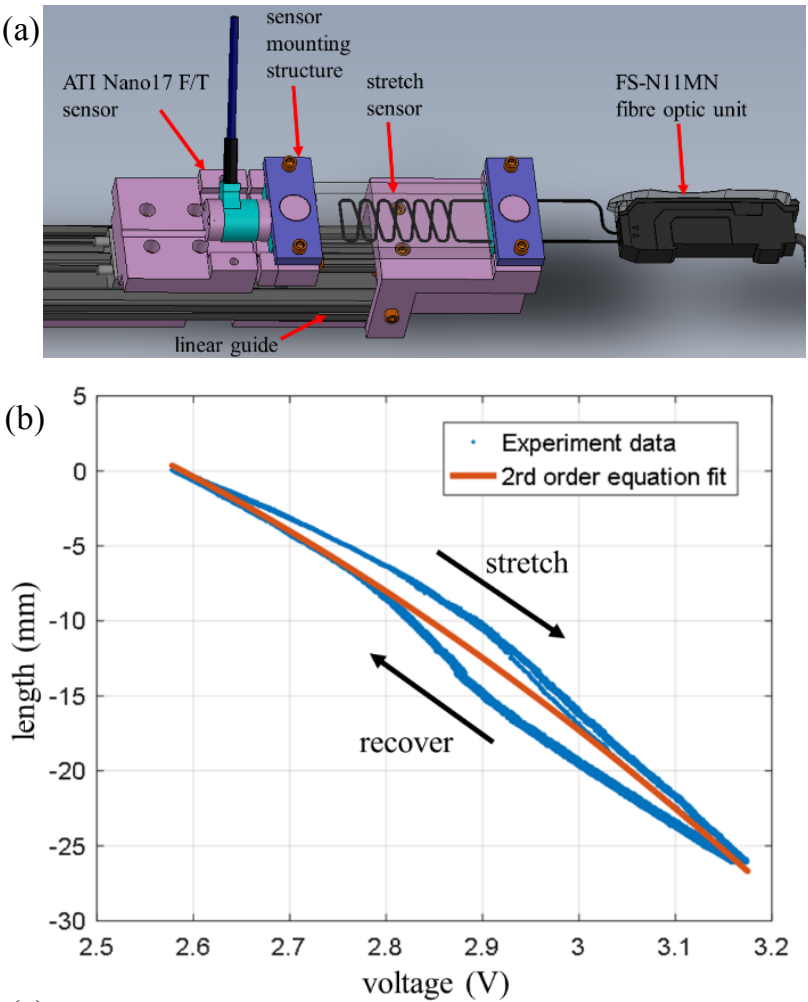

(c)

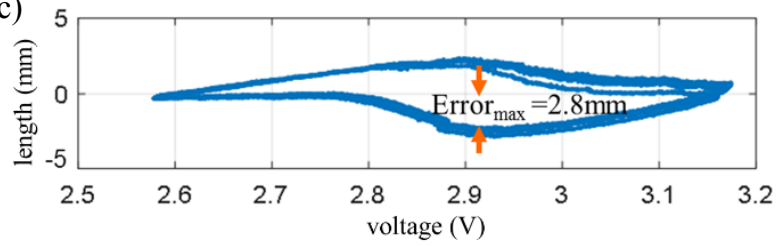

Figure 6. (a) The experimental calibration set-up, (b) the sensor length-voltage relationship (over 5 cycles) and respective $2^{\text {nd }}$ order fit to the data, and (c) the error values.

D) Stiffness test and impact of the sensor integration on linearity of the soft structure

We have performed a force test to study the stiffness properties of the stretch sensor and the possible impact of sensor integration to the linearity of the elastic silicone material. (a)

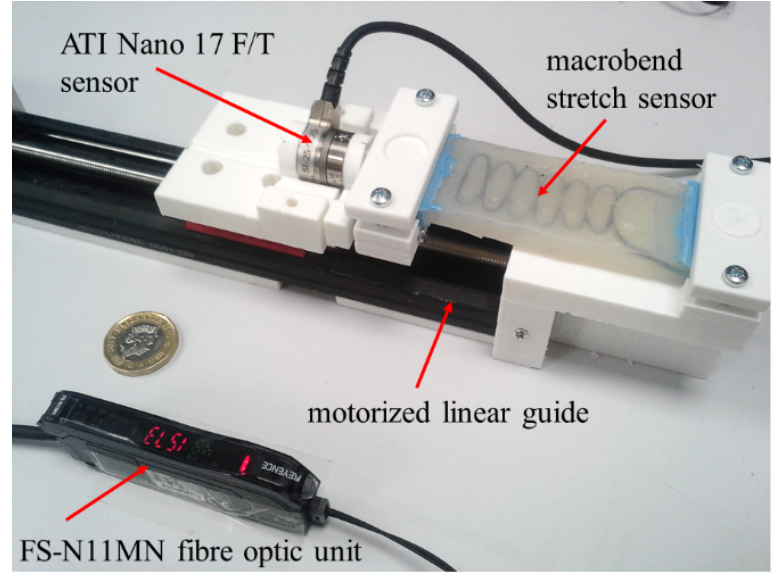

(b)

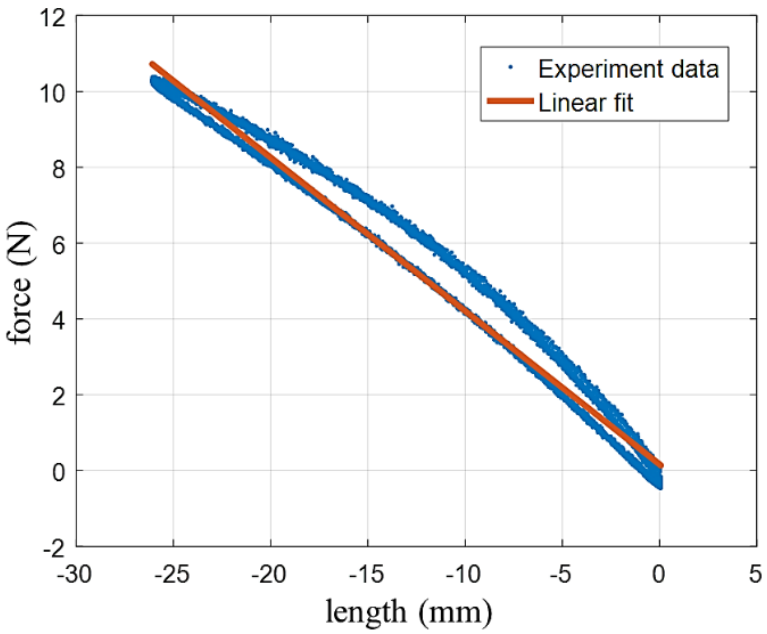

Figure 7. Sensor's impact on linearity of the silicone structure: (a) the experimental force sensing setup, and (b) experimental results. Note that a Nano17 F/T sensor is linked in serial with the macrobend stretch sensor.

In this experiment, a Nano17 force sensor fixed to the moving platform of a motorized linear guide was serially linked to the sensor. This experimental arrangement, shown in Figure 7(a), provided position and force information during the extension and compression of the sensor (Figure 7(b)).

The experimental results show that the maximum stiffness coefficient of the stretch sensor is approximately $0.4 \mathrm{~N} / \mathrm{mm}$ and it demonstrates a near-linear behavior. Therefore, it can be concluded that, the integration of the optical fibre into the silicone structure has a negligible impact on the linearity of the structure.

\section{CONCLUSIONS AND FUTURE WORKS}

In this study, low profile stretch sensors created from highly flexible and light weight optical fibres exploiting bendinduced light loss sensing principle. The light outputs passing through the optical fibres varies due to macrobend loss, which is a function of the fibre bend radius as well as the number of repeated turns. This study revealed that the chosen bend radius $r$ has a far greater impact on the measured light 
intensity values than the number of turns $\mathrm{N}$ (if $\mathrm{N} \geq 5$ ). Hence, taking into account that the bend radius is the only significantly influencing design parameter, macrobend stretch sensors with $\mathrm{N} \geq 5$ were developed to create a low cost and low profile solution to stretch sensing in soft robots.

The sensor is designed so that the optical fibre originate from the base and extend to the tip of the sensor structure in a serpentine pattern, then loop back to the base to allow placing all required opto-electronics at one end of the sensor. This loopback optical fibre system is inherently safe as no electrical current is required to pass through the sensor's soft structure, and is suitable for modular as well as array implementations. Since, in the proposed sensor, a single piece optical fibre creates a closed optical loop between the emitter and the detector, it is immune to the external lighting conditions. Moreover, the ability of the macrobend stretch sensors to flexibly adapt their configuration allows preserving the inherent softness and compliance of soft actuation systems they are coupled with.

The future work will consider improvements on the fabrication process of the sensor enabling creation of identical sensor components.

\section{REFERENCES}

[1] M. A. Delph, S. A. Fischer, P. W. Gauthier, C. H. M. Luna, E. A. Clancy, and G. S. Fischer, "A soft robotic exomusculature glove with integrated sEMG sensing for hand rehabilitation," in IEEE International Conference on Rehabilitation Robotics, 2013, pp. 1-7.

[2] P. Polygerinos, Z. Wang, K. C. Galloway, R. J. Wood, and C. J. Walsh, "Soft robotic glove for combined assistance and at-home rehabilitation," Rob. Auton. Syst., vol. 73, pp. 135-143, 2015.

[3] J. Rossiter, E.Knoop, Y.Nakamura, 2017, 'Affective Touch and Low Power Artificial Muscles for Rehabilitative and Assistive Wearable Soft Robotics'. in: Wearable Robotics: Challenges and Trends: Proceedings of the 2nd International Symposium on Wearable Robotics, WeRob2016, October 18-21, 2016, Segovia, Spain. Springer, pp. 101-106.

[4] A. Firouzeh, J. Paik, Development of Essential Components for Soft Wearable Technologies. EPFL, Lausanne, 2017.

[5] J. Kim, A. Alspach, and K. Yamane, "3D printed soft skin for safe human-robot interaction," in Proc. IEEE/RSJ Int. Conf. Intell. Robots Syst. (IROS'15). IEEE, 2015, pp. 2419-2425.

[6] P.Sareh and M.Kovac, "Mechanized creatures", Science 355 (6332), 1379. [doi: 10.1126/science.aam9075].

[7] J.Yi, X.Chen, and Z.Wang, A Three-Dimensional-Printed Soft Robotic Glove With Enhanced Ergonomics and Force Capability, IEEE Robotics and Automation Letters, VOL. 3, NO. 1, JANUARY 2018.

[8] Y-L.Park, B.Chen, N.O. Pérez-Arancibia, D.Young, L.Stirling, R.J.Wood, E.Goldfield, R.Nagpal, Design and control of a bio-inspired soft wearable robotic device for ankle-foot rehabilitation 2014 Bioinspir. Biomim. 9016007.

[9] S.Sareh, Y.Noh, M.Li, T.Ranzani, H.Liu, K.Althoefer, Macro-bend optical sensing for pose measurement in soft robot arms, Smart Mater. Struct. 24 125024, 2015.

[10] D.M.Hannan, and D.I.Walker, "Vision based shape estimation for continuum robots" Proc. Of the 2003 IEEE ICRA (Taipei, Taiwan) pp. $3449-3454,2003$

[11] C.A. Nafis, V.Jensen, L.Beauregard, P.T.Anderson (2006) "Method for estimating dynamic EM tracking accuracy of Surgical Navigation tools", SPIE Medical Imaging Proceedings, 2006.

[12] V.S.Vibhute, and A.Kshirsaga, Identification of Hysteresis and Relaxation Parameters in Stretch Sensor, 10.1214/IJAEEE/25, 2012.
[13] B.O'Brien, T.Gisby, I.Anderson "Stretch sensors for human body motion" Proc. SPIE 9056, Electroactive Polymer Actuators and Devices (EAPAD) 2014, 905618,2014.

[14] C.S. Liu, G.W.Chou, X.M.Liang, P.G.Reinhall, and W.C.Wang "Design of a Multi-layered Optical Bend Loss Sensor for Pressure and Shear Sensing" Proc. of SPIE Smart Structures and Materials \& Nondestructive Evaluation and Health Monitoring, 5768-36, 2007.

[15] S.Sareh, K.Althoefer, M.Li, Y.Noh, F.Tramacere, P.Sareh, B.Mazzolai, M.Kovac "Anchoring like octopus: biologically inspired soft artificial sucker" Journal of the Royal Society Interface, 14:135, 2017. [DOI: 10.1098/rsif.2017.0395]

[16] S. Sareh, Y. Noh, T.Ranzani, H.Wurdemann, H.Liu, K.Althoefer, A $7.5 \mathrm{~mm}$ Steiner chain fiber-optic system for multi-segment flex sensing, IEEE/RSJ International Conference on Intelligent Robots and Systems (IROS), 2336-2341, 2015.

[17] Y.Noh, S.Sareh, H.Wudermann, H. Liu, K. Althoefer, Three Axial Fiber-Optic Body Force Sensor for Flexible Manipulators, Sensors11652-2015, 2015.

[18] S.Sareh, A.Jiang, A.Faragasso, Y.Noh, T.Nanayakkara, P.Dasgupta, L.Seneviratne, H.Wurdemann, K.Althoefer "Bio-Inspired Tactile Sensor Sleeve for Soft Surgical Manipulators" IEEE International Conference on Robotics and Automation (ICRA), Hong Kong, China, 2014.

[19] H.Wurdemann, S.Sareh, A.Shafti, Y.Noh, A.Faragasso, H.Liu, S.Hirai, K.Althoefer "Embedded electro-conductive yarn for shape sensing of soft robotic manipulators",Engineering in Medicine and Biology Conference (EMBC), 2015.

[20] T.Allsop, M.Dubov, A.Martinez, F.Floreani, I. Khrushchev, D.Webb, and I.Bennion, Long period grating directional bend sensor based on asymmetric index modification of cladding, Electronics Letters, vol. 41, no. 2, 2005.

[21] J.Feng, Y.Zhao, X-W.Lin, W.Hu, F.Xu, Y.Q.Lu "A Transflective Nano-Wire Grid Polarizer Based Fibre-Optic Sensor", Sensors, 11(3): 2488-2495, 2011.

[22] R.J.Roesthuis, S.Janssen, S.Misra "On using an array of fibre Bragg grating sensors for closed-loop control of flexible minimally invasive surgical instruments," IEEE/RSJ International Conference on in Intelligent Robots and Systems (IROS), pp. 2545-2551, 2013.

[23] X.Zhang, J.J.Max, X.Jiang, L.Yu, H.Kassi “Experimental investigation on optical spectral deformation of embedded FBG sensors", Proc. SPIE 6478, Photonics Packaging, Integration, and Interconnects VII, 647808, 2007.

[24] A.J.Jay. "An overview of macrobending and microbending of optical fibres", White Paper WP1212, Corning, Dec 2010.

[25] Silva, A.S, Catarino, A., Correia, M.V., Frazao, O. (2013) Design and characterization of a wearable macrobending fibre optic sensor for human joint angle determination, Opt. Eng. 52(12), 126106.

[26] M.A.Zawawi, S.O'Keeffe, E.Lewis, "Intensity-modulated fibre optic sensor for health monitoring applications: a comparative review", Sensor Review, Vol. 33 Iss: 1, pp.57, 2013.

[27] W.C.Wang, W.Ledoux, B.Sangeorzan, P.Reinhall "A shear and plantar pressure sensor based on fibre-optic bend loss," Journal of Rehabilitation Research and Development, 42(3), 315-326, 2005.

[28] P.Wang, Y.Semenova, Q.Wu, G.Farrell "A fibre-optic voltage sensor based on macrobending structure" Optics \& Laser Technology 43 (2011) 922-925, 2011.

[29] W. Belardi, and J.C.Knight "Hollow antiresonant fibres with low bending loss" Opt. Express 22, 10091-10096, 2014.

[30] R.J.Hoss, E.A.Lacy "Fiber Optics" 2nd Edition, ISBN: 0-13-3212416, 1993. 